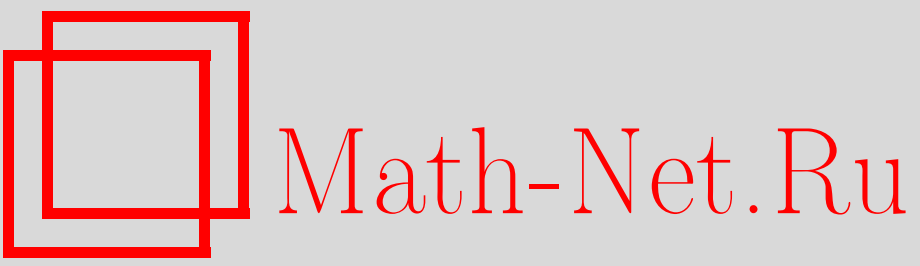

Л. А. Сараев, Е. В. Михайлова, В. Н. Никишов, Математическая модель построения доверительных оценок скрытых источников инвестиционного дохода, Вестн. Сам. гос. техн. ун-та. Сер. Физ.-мат. науки, 2009, выпуск 1(), 182-190

DOI: https://doi.org/10.14498/vsgtu674

Использование Общероссийского математического портала Math-Net.Ru подразумевает, что вы прочитали и согласны с пользовательским соглашением

http://www. mathnet.ru/rus/agreement

Параметры загрузки:

IP : 54.198 .55 .26

26 апреля 2023 г., 07:48:01 
УДК 519.865.7+51-7:330.322.011

\section{МАТЕМАТИЧЕСКАЯ МОДЕЛЬ ПОСТРОЕНИЯ ДОВЕРИТЕЛЬНЫХ ОЦЕНОК СКРЫТЫХ ИСТОЧНИКОВ ИНВЕСТИЦИОННОГО ДОХОДА}

\section{Л. А. Сараев, В.Н. Никишов, Е.В. Михайлова}

Самарский государственный университет, 443011, Самара, ул. ак. Павлова, 1.

E-mails: saraev_leo@mail.ru, TSh-sea05@yandex.ru, milena82@yandex.ru

Предложена математическая модель построения доверительньх оченок скрытых источников инвестиционного дохода. Получены выражсния для расчёта среднего времени задержки межсу поступлением и расходованием средств предприятия и прогнозных значений нормы прибыльности для группы производимых хозяйствующим субъектом товаров на следующий период времени.

Ключевые слова: оценка параметров распределения, инвестищионный доход.

Введение. Хозяйствующий субъект, производящий товары (выполняющий работы или оказывающий услуги), осуществляет закупки материалов, оплату труда рабочих и служащих, иные производственные и непроизводственные затраты. После реализации товара (выполненных работ, оказанных услуг) хозяйствующий субъект получает денежные средства, которые вкладывает в продолжение производства. Когда объём поступлений от реализации превышает объём расходов, прибыль положительна. В этом случае предприятие (фирма) имеет инвестиционный доход. Однако возможны ситуации, когда объёмы поступлений и расходов совпадают. Каким образом в этом случае формируются инвестиционные средства? В работе предлагается математическая модель построения доверительных оценок скрытых источников инвестиционного дохода.

Оценка инвестиционного дохода бесприбыльного предприятия. Допустим, во-первых, что фактическая доходность предприятия равна фактической себестоимости, а во-вторых, что процессы производства товаров или услуг и процессы поступления средств от их реализации являются непрерывными. Обозначим $r(t)$ - скорость расходов, $w(t)$ - скорость поступлений. Тогда на отрезке времени $[0, t]$ общий объём расходов $R$ и поступлений $W$ определяются формулами:

$$
R(t)=\int_{0}^{t} r(t) d t, \quad W(t)=\int_{0}^{t} w(t) d t,
$$

их связь при указанных предположениях - соотношением

$$
R(t)=W(t)
$$

Сараев Леонид Александрович-зав. кафедрой математики, информатики и математических методов в экономике; д.ф.-м.н., профессор.

Никишов Виктор Николаевич - доцент кафедры математики, информатики и математических методов в экономике; к.ф.-м.н., дочент.

Михайлова Елена Владимировна - ст. преподаватель кафедры математики, информатики и математических методов в экономике. 
Очевидно, что при указанных предположениях нет никакой основы для создания инвестиционного дохода:

$$
\Delta I=i \int_{0}^{t}(w(t)-r(t)) d t=0,
$$

где $i$ - норма доходности.

Однако на практике предприятия получает инвестиционный доход. Формула (1) неверна, так как при расчёте не учтена длительность производственного процесса и задержка между временем поступления и расходования средств. Такая задержка выплат имеет, как правило, стохастический характер, особенно заметный, например, в работе страховых организаций, когда объём поступающей нетто-премии в любой момент времени равен объёму выплат, но неизвестно, в какой момент времени это произойдет. Пусть $\tau$-среднее время задержки между поступлением и расходованием средств (заметим, что при равномерном расходовании средств $\tau=T / 2$ ). Это время зависит от характера конкретного производства и срока его осуществления $T$. Тогда имеет место соотношение

$$
r(t)=w(t-\tau) .
$$

Элемент расходов $d r=r(t) d t=w(t-\tau) d t$ определяется поступлением доходов, полученных раньше на время $\tau$ (заметим, что здесь $\tau$ также выражает среднее время существования средств предприятия на счетах). Инвестиционный доход за отчетный период $T$ :

$$
\Delta I(T)=i \int_{0}^{T} w(t)[\eta(x)-\eta(t-\tau)] d t=i w_{0}\left[T-\int_{\tau}^{T} d t\right]=i w \tau=i W \frac{\tau}{T} .
$$

В общем случае значение (2) является максимальным значением возможного инвестиционного дохода для бесприбыльного предприятия.

Предполагая равномерное распределение среднего времени выплат задержки, получим оценку инвестиционного дохода для бесприбыльного предприятия:

$$
\Delta I=\frac{i W \tau}{T}=\frac{i W}{2} .
$$

Пусть $\alpha$ - часть средств от дохода предприятия $D$, которая сразу идет на оплату счетов (на практике $\alpha$ составляет от $10 \%$ до $30 \%$ ). Следовательно, часть дохода в размере $(1-\alpha) \cdot D$ будет израсходована постепенно в производственном процессе. В этом случае $W=(1-\alpha) \cdot D$. Например, при доходе за период $T=1$ год в размере $D, \alpha=0,2$ и $i=10 \%$ имеем максимально возможный инвестиционный доход в размере $I D=0,8 \cdot 0,1 \cdot D=0,08 \cdot D$, т. е. $8 \%$ от годовой выручки.

Таким образом, скрытым источником инвестиционного дохода предприятия является временная распределённость производственного процесса, когда получаемые доходы можно использовать для его инвестирования в течение среднего времени задержки.

Как уже отмечалось, оценка среднего времени зависит от специфики деятельности предпринимателя. Пусть производственный цикл длится $T_{0}$, тогда 
за год возможно осуществление $n=T / T_{0}$ циклов. Средний размер денежных средств на счете предприятия определяется формулой

$$
S \approx(1-\alpha) D \cdot \tau \frac{T}{T_{0}}
$$

а среднее время задержки между поступлением и расходованием соотношением

$$
\tau=\min \left\{\frac{T_{0}}{2}, \frac{S T_{0}}{(1-\alpha) D T}\right\} .
$$

Для такой специфической деятельности, как страхование, среднее время задержки есть стохастическая величина, определяемая вероятностными методами. В этом случае распределение времени задержки $\xi$ можно описать функцией

$$
F_{\xi}(t)=P(\xi<t)=1-\exp \left(-\frac{w}{T} t\right), \quad F_{\xi}(T)=Q, \quad w=-\ln (1-Q),
$$

где $Q$ - есть вероятность реализации риска по договорам.

Для нахождения среднего времени $\tau=E[\xi]$ имеем условную функцию распределения:

$$
\Phi_{\xi}(t)=P(\xi<t \mid t<T)=1-\frac{\exp \left(-\frac{w}{T} t\right)}{1-\exp (-w)} .
$$

Используя (3), находим:

$$
\begin{gathered}
\frac{E[\xi]}{T}=\frac{\xi_{0}}{T}=\frac{\tau}{T}=\frac{1}{w}-\frac{1-Q}{Q}, \\
\frac{E\left[\xi^{2}\right]}{T^{2}}=\frac{2}{w^{2}}-\frac{1-Q}{Q}\left(\frac{2}{w}+1\right), \\
\frac{\sigma^{2}(\xi)}{T^{2}}=\frac{1}{w^{2}}-\frac{(1-Q)^{2}}{Q^{2}}-\frac{1-Q}{Q} .
\end{gathered}
$$

На рисунке представлен график зависимости нормированного среднего времени, представленного формулой (4), и нормированного среднеквадратичного отклонения, представленного формулой (5), от значения вероятности $Q$.

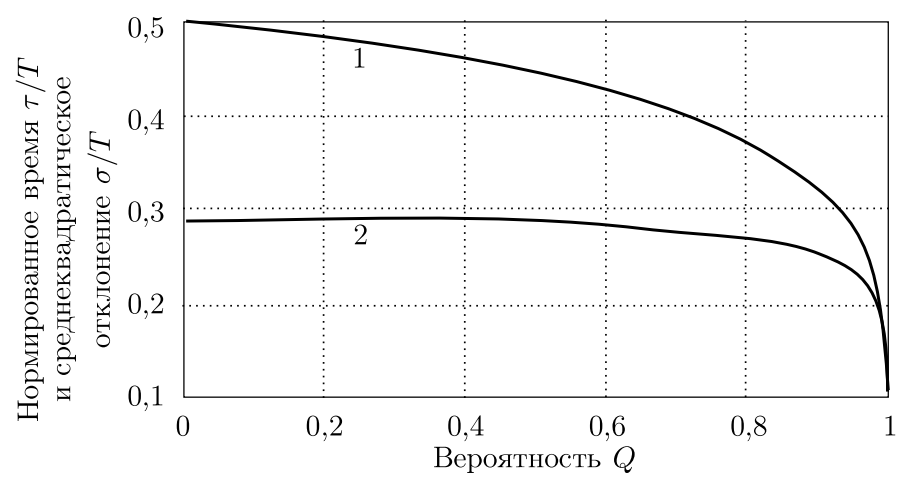

1 - зависимость нормированного среднего времени задержки; между поступлением и расходованием средств от вероятности

2 - зависимость нормированного среднеквадратичного отклонения от вероятности. 
Оценка инвестиционного дохода предприятия, получающего прибыль. Вторым источником получения инвестиционного дохода является прибыль - разность между доходами и затратами (себестоимостью продукции).

Рассмотрим производственную деятельность предприятия - выпуск группы продукции за ряд лет. Будем понимать под производственной матрицей таблицу, в которой по строкам расположены однородные партии товаров (работ, услуг) по годам, а её элементами являются затраты на производство $S_{i j}$ и прибыль от реализации - $D_{i j}$, где $i=1,2, \ldots n$ - количество групп (номенклатура) товаров; $j=1,2, \ldots, m-$ количество лет наблюдений.

Разобьём всю совокупность производимой продукции (в дальнейшем портфель) на группы согласно таблице.

\section{Производственная матрица предприятия}

\begin{tabular}{c|c|c|c}
\hline$S_{11}, D_{11}$ & $S_{12}, D_{12}$ & $\ldots$ & $S_{1 m}, D_{1 m}$ \\
\hline$S_{21}, D_{21}$ & $S_{22}, D_{22}$ & $\ldots$ & $S_{2 m}, D_{2 m}$ \\
\hline$\ldots$ & $\ldots$ & $\ldots$ & $\ldots$ \\
\hline$S_{n 1}, D_{n 1}$ & $S_{n 2}, D_{n 2}$ & $\ldots$ & $S_{n m}, D_{n m}$ \\
\hline
\end{tabular}

Величина $X_{i j}=D_{i j} / S_{i j}$ - норма прибыльности по данной группе $i$ в год наблюдения $j$.

Пусть индекс $(+)$ означает суммирование и

$$
\begin{gathered}
S_{i+}=\sum_{j=1}^{m} S_{i j}, \quad S_{+j}=\sum_{i=1}^{n} S_{i j}, \quad S_{++}=\sum_{i=1}^{n} \sum_{j=1}^{m} S_{i j}, \\
D_{i+}=\sum_{j=1}^{m} D_{i j}=\sum_{j=1}^{m} X_{i j} S_{i j}, \quad D_{+j}=\sum_{i=1}^{n} D_{i j}=\sum_{i=1}^{n} X_{i j} S_{i j}, \\
D_{++}=\sum_{i=1}^{n} \sum_{j=1}^{m} D_{i j}=\sum_{i=1}^{n} \sum_{j=1}^{m} X_{i j} S_{i j} .
\end{gathered}
$$

Тогда

$$
\begin{gathered}
X_{i+}=\frac{D_{i+}}{S_{i+}}=\frac{1}{S_{i+}} \sum_{j=1}^{m} D_{i j}=\frac{1}{S_{i+}} \sum_{j=1}^{m} X_{i j} S_{i j}, \\
X_{+j}=\frac{D_{+j}}{S_{+j}}=\frac{1}{S_{+j}} \sum_{i=1}^{n} D_{i j}=\frac{1}{S_{+j}} \sum_{i=1}^{n} X_{i j} S_{i j}, \\
X_{++}=\frac{D_{++}}{S_{++}}=\frac{1}{S_{++}} \sum_{i=1}^{n} \sum_{j=1}^{m} D_{i j}=\frac{1}{S_{++}} \sum_{i=1}^{n} \sum_{j=1}^{m} X_{i j} S_{i j} .
\end{gathered}
$$

На первом этапе проверяется гипотеза о равенстве средних значений по группам с помощью дисперсионного анализа (analysis of variance, ANOVA) [1]. Если гипотеза о равенстве всех средних неверна, это означает, что изменчивость средних $X_{i+}$ по группам относительно среднего $X_{++}$по всему классу 
(портфелю) оказывается больше, чем это можно было бы ожидать на основании измерения изменчивости внутри групп. Вычисляя внешнюю и внутреннюю суммы квадратов и построив дисперсионное отношение, можно осуществить проверку критерием Фишера [1]. Если гипотеза о равенстве средних принимается, то нет оснований считать портфель (группу) неоднородным, но обычно это не так. Если гипотеза отвергается, то средние по группам различны. При этом можно считать средние неизвестными числами и пытаться обнаружить закономерность, которой они подчиняются, например, строя регрессию на данные наблюдений.

На практике часто возникает необходимость вычисления убытков или дохода для группы контрактов, по которым имеется некоторый ряд значений по предыдущим периодам.

В доверительной теории [1-3] предлагается модель вида

$$
X_{i j}=m+\Omega_{i}+\Omega_{i j}
$$

где $m$ - среднее по портфелю; $\Omega_{i}$ - случайная величина, характеризующая отклонение от общего среднего, обусловленное принадлежностью к группе (называется структурным распределением, так как описывает структуру портфеля); $\Omega_{i j}$ - случайная величина, характеризующая изменчивость по годам группы под номером $i$.

Характеристики величин $\Omega_{i}$ и $\Omega_{i j}$ представлены соотношениями

$$
\begin{gathered}
E\left[\Omega_{i}\right]=0, \quad \operatorname{Var}\left[\Omega_{i}\right]=E\left[\Omega_{i}^{2}\right]=a^{2}, \quad E\left[\Omega_{i} \Omega_{j}\right]=a^{2} \delta_{i j} \\
E\left[\Omega_{i k}\right]=0, \operatorname{Var}\left[\Omega_{i j}\right]=E\left[\Omega_{i j}^{2}\right]=\frac{s^{2}}{S_{i j}}
\end{gathered}
$$

где $m, a^{2}, s^{2}$ - структурные параметры.

В случае, когда в каждой ячейке находится $N_{i j}$ товаров (контрактов, работ, услуг), а величины $D_{i j}=\sum_{k=1}^{N_{i j}} D_{i j k}$ и $S_{i j}=\sum_{k=1}^{N_{i j}} S_{i j k}$, получаем

$$
X_{i j}=\frac{D_{i j}}{S_{i j}}=\frac{\sum_{k=1}^{N_{i j}} D_{i j k}}{\sum_{l=1}^{N_{i j}} S_{i j k}}=\frac{1}{S_{i j}} \sum_{k=1}^{N_{i j}} S_{i j k} X_{i j k} .
$$

Если группа однородна (или близка к ней), то $S_{i j k} \approx d_{i j}, \operatorname{Var}\left[X_{i j k}\right]=\sigma_{i j}^{2}$, и тогда $\operatorname{Var}\left[X_{i j}\right]=\frac{d_{i j}^{2}}{N_{i j}}=\frac{d_{i j}^{3}}{N_{i j} d_{i j}}=\frac{s^{2}}{S_{i j}}$, что соответствует выражению (8), т. е. выражение (9) можно распространить на слабо-неоднородные группы.

Учитывая (6), (7) и (8), имеем

$$
E\left[X_{i j}\right]=m ; \quad \operatorname{Var}\left[X_{i j}\right]=E\left[\left(X_{i j}-m\right)^{2}\right]=a^{2}+\frac{s^{2}}{S_{i j}} .
$$

Основной задачей является нахождение прогнозных значений нормы прибыльности для каждой группы на следующий период времени, т. е. $X_{u, m+1}$, где $u=1,2, \ldots, n$. В целях сокращения записи введем обозначение $Z^{(u)}=$ $=X_{u, m+1}$. Условие несмещённости имеет вид $E\left[Z^{(u)}\right]=m$. 
Будем искать $Z^{(u)}$ в виде суммы по всем известным значениям $X_{i j}$ с некоторыми коэффициентами $\alpha_{i j}^{(u)}$, подлежащими определению:

$$
Z^{(u)}=\sum_{i=1}^{n} \sum_{j=1}^{m} \alpha_{i j}^{(u)} X_{i j} .
$$

Тогда условие несмещённости примет вид

$$
E\left[\sum_{i, j} \alpha_{i j}^{(u)} X_{i j}\right]=m
$$

Построим лагранжиан вида

$$
L=\frac{1}{2} E\left[\sum_{i, j}^{\alpha_{i j}^{(u)}} X_{i j}-Z^{(u)}\right]^{2}+\alpha_{0}^{(u)} E\left[\sum_{i j}^{\alpha_{i j}^{(u)} X_{i j}-m}\right]
$$

Дифференцируя (12) по $\alpha_{0}^{(u)}, \alpha_{r s}^{(u)}$, получим систему уравнений:

$$
\left\{\begin{array}{l}
\sum_{i, j} \alpha_{i j}^{(u)} E\left[X_{i j}\right]=m, \\
\sum_{i, j} \alpha_{i j} E\left[X_{i j} X_{r s}\right]+\alpha_{0}^{(u)} E\left[X_{r s}\right]=E\left[Z^{(u)} X_{r s}\right], \\
r=1,2, \ldots, n ; s=1,2, \ldots, m .
\end{array}\right.
$$

Учитывая (11), имеем

$$
\left\{\begin{array}{l}
\sum_{i, j} \alpha_{i j}^{(u)}=1, \\
\sum_{i, j} \alpha_{i j} E\left[X_{i j} X_{r s}\right]+\alpha_{0}^{(u)} E\left[X_{r s}\right]=E\left[Z^{(u)} X_{r s}\right], \\
r=1,2, \ldots, n ; s=1,2, \ldots, m
\end{array}\right.
$$

где $E\left[X_{i j} X_{r s}\right]=E\left[\left(m+\Omega_{i}+\Omega_{i, j}\right)\left(m+\Omega_{r}+\Omega_{r s}\right)\right]=m^{2}+\delta_{i r} a^{2}+\delta_{i r} \delta_{j s} \frac{s^{2}}{S_{r s}}$, $E\left[X_{r s}\right]=m, E\left[Z^{(u)} X_{r s}\right]=E\left[\left(m+\Omega_{u}+\Omega_{u J+1}\right)\left(m+\Omega_{r}+\Omega_{r s}\right)\right]=m^{2}+\delta_{u r} a^{2}$.

Из второго уравнения (13) получаем

$$
\alpha_{r+}^{(u)}+\frac{\widehat{s}^{2} \alpha_{r s}^{(u)}}{S_{r s}}=\delta_{u r}-b^{(u)},
$$

где $b^{(u)}=\alpha_{0}^{(u)} \frac{m}{a^{2}}$ и $\widehat{s}^{2}=\frac{s^{2}}{a^{2}}$.

Перепишем (14) в виде

$$
\alpha_{r+}^{(u)} S_{r s}+\widehat{s}^{2} \alpha_{r s}^{(u)}=\left(\delta_{u r}-b^{(u)}\right) S_{r s} .
$$


Суммируя по $s(15)$, получим

$$
\alpha_{r+}^{(u)}\left(S_{r+}+\widehat{s}^{2}\right)=\left(\delta_{u r}-b^{(u)}\right) S_{r+}, \quad \alpha_{r+}^{(u)}=\left(\delta_{u r}-b^{(u)}\right) \gamma_{r},
$$

где $\gamma_{r}=\frac{S_{r+}}{S_{r++\widehat{s}^{2}}}$.

Суммируя (16) по $r$ и учитывая первое уравнение (13), находим

$$
b^{(u)}=-\frac{\left(1-\gamma_{u}\right)}{\gamma}
$$

где $\gamma_{u}$-коэффициент достоверности к статистике группы (чем ближе $\gamma_{u}$ к единице, тем большее значение имеет статистика группы); $\gamma=\sum_{u=1}^{n} \gamma_{u}$.

Подставляя выражение для (17) в (16), получим

$$
\alpha_{r+}^{(u)}=\gamma_{r}\left(\delta_{u r}+\frac{1-\gamma_{u}}{\gamma}\right)
$$

Из (15) с учётом (18) находим

$$
\alpha_{r s}^{(u)}=\frac{S_{r s}}{S_{r+}} \gamma_{r}\left(\delta_{u r}+\frac{1-\gamma_{u}}{\gamma}\right) .
$$

Таким образом, получаем прогнозное значение на следующий период времени $t=m+1$ с использованием всей статистки по портфелю:

$$
\begin{aligned}
Z^{(u)}=X_{u, J+1}=\sum_{r, s} \alpha_{r s}^{(u)} X_{r s}=\sum_{r, s} \frac{S_{u s}}{S_{u+}} \gamma_{r}\left(\delta_{u r}+\frac{1-\gamma_{u}}{\gamma}\right) X_{r s}= & \\
& =\gamma_{u} X_{u+}+\left(1-\gamma_{u}\right) \sum_{r=1}^{n} \frac{\gamma_{r}}{\gamma} X_{r+}
\end{aligned}
$$

Применение выражения (20) для практических вычислений требует предварительного получения оценок структурных параметров $a^{2}$ и $s^{2}$.

Для оценки дисперсионного параметра $s^{2}$ рассмотрим выражение вида

$$
\left\langle s^{2}\right\rangle=\frac{1}{n(m-1)} \sum_{i, j} S_{i j}\left(X_{i j}-X_{i+}\right)^{2} .
$$

В этом случае, с учётом того, что

$$
\begin{gathered}
E\left[X_{i j}^{2}\right]=E\left[\left(m+\Omega_{i}+\Omega_{i j}\right)^{2}\right]=m^{2}+a^{2}+\frac{s^{2}}{S_{i j}} \\
E\left[X_{i j} X_{i+}\right]=m^{2}+a^{2}+\frac{s^{2}}{S_{i+}}, \quad E\left[X_{i+}^{2}\right]=m^{2}+a^{2}+\frac{s^{2}}{S_{i+}},
\end{gathered}
$$

имеем несмещенную оценку: $E\left[\left\langle s^{2}\right\rangle\right]=s^{2}$. 
Рассмотрим выражение вида

$$
B=\sum_{i=1}^{n} S_{i+}\left(X_{i+}-X_{++}\right)^{2}=\sum_{i=1}^{n} S_{i+} X_{i+}^{2}-S_{++} X_{++}^{2} .
$$

С учётом того, что

$$
E\left[X_{i+}^{2}\right]=m^{2}+a^{2}+\frac{s^{2}}{S_{i+}}, \quad E\left[X_{++}^{2}\right]=m^{2}+a^{2} \frac{1}{S_{++}^{2}} \sum_{i} S_{i+}^{2}+\frac{s^{2}}{S_{++}},
$$

получим

$$
E[B]=a^{2} S_{++} K+s^{2}(n-1) .
$$

Тогда в качестве оценки для $a^{2}$ имеем выражение

$$
\left\langle a^{2}\right\rangle=\frac{B-s^{2}(n-1)}{S_{++}(1-K)}
$$

где $K=1-\frac{1}{S_{++}^{2}} \sum_{i=1}^{n} S_{i+}^{2}$.

Выражение $(20)$ для $X_{i, m+1}^{(u)}$ и выражения (21) и (22) для определения оценок структурных параметров полностью решают задачу.

Приведём также оценку величины $m=E\left[X_{i j}\right]$ :

$$
\langle m\rangle=X_{++}=\frac{1}{S_{++}} \sum_{i=1}^{n} \sum_{j=1}^{m} S_{i j} X_{i j}
$$

хотя в данном подходе непосредственно она не используется.

Заключение. Таким образом, получено, во-первых, выражение (4) для среднего времени задержки между поступлением и расходованием средств хозяйствующего субъекта, являющегося источником инвестиционного дохода бесприбыльного предприятия; во-вторых, выражения для прогнозного значения нормы прибыльности (20) и оценки структурных параметров (21) и (22), в случае, когда разность доходов и расходов от производства (выполненных работ, оказанных услуг) положительна.

\section{БИБЛИОГРАФИЧЕСКИЙ СПИСОК}

1. Крамер Г. Математические методы статистики. - М.: Мир, 1975. - 648 с.

2. Каас P., Гувертс М., Дэне Ж., Денут М. Современная актуарная теория риска. - М.: Янус-К, 2007. - 372 с.

3. Мак T. Математика рискового страхования. - М.: Олимп-Бизнес, 2005. - 432 с.

4. Штрауб Э. Актуарная математика имущественного страхования. - М.: Крокус-Т, 1990. $147 \mathrm{c}$. 
MSC: 62P20, 91B28

\section{MATHEMATICAL MODEL OF CONSTRUCTION OF CONFIDENTIAL ESTIMATIONS OF THE HIDDEN SOURCES OF INVESTMENT INCOME}

\section{A. Saraev, V.N. Nikishov, E. V. Mikhailova}

Samara state university

1, Ak. Pavlova st., Samara, 443011.

E-mails: saraev_leo@mail.ru, TSh-sea05@yandex.ru, milena82@yandex.ru

Mathematical model of construction of confidential estimations of the hidden sources of investment income was studied. Expressions for calculation of average time of a delay between receipt and expenditure of financial stock of an enterprise and forecast values of profitability norms for a group of the goods produced by a managing subject for the following period of time were obtained.

Key words: estimation of parameters of distribution, the investment income.

Original article submitted 04/XII/2008; revision submitted 09/II/2009.

Saraev Leonid Aleksandrovich, Dr. Sci. (Phys. \& Math.), Prof., Head of Dept. of Mathematics, Computer Science and Mathematical Methods in Economy.

Nikishov Victor Nikolaevich, Ph. D. (Phys. ES Math.), Ass. Prof., Dept. of Mathematics, Computer Science and Mathematical Methods in Economy.

Mikhailova Elena Vladimirovna, Senior Lecture, Dept. of Mathematics, Computer Science and Mathematical Methods in Economy. 